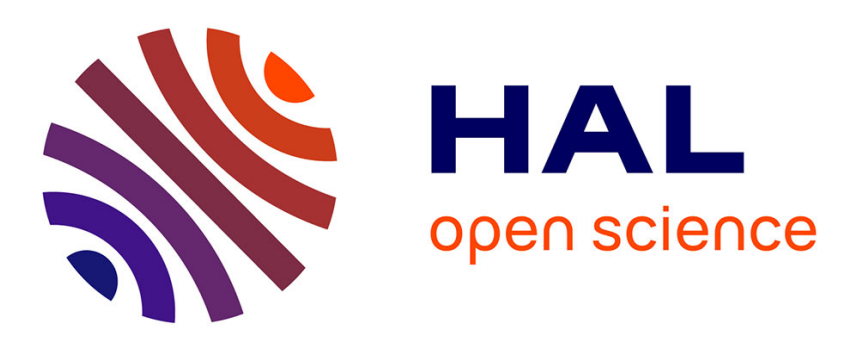

\title{
Selection Rules for Point-Defect Anelastic Relaxations : Classical versus Quantum Mechanical
}

\author{
W. Johnson
}

\section{To cite this version:}

W. Johnson. Selection Rules for Point-Defect Anelastic Relaxations: Classical versus Quantum Mechanical. Journal de Physique IV Proceedings, 1996, 06 (C8), pp.C8-27-C8-30. 10.1051/jp4:1996803 . jpa-00254520

\section{HAL Id: jpa-00254520 https://hal.science/jpa-00254520}

Submitted on 1 Jan 1996

HAL is a multi-disciplinary open access archive for the deposit and dissemination of scientific research documents, whether they are published or not. The documents may come from teaching and research institutions in France or abroad, or from public or private research centers.
L'archive ouverte pluridisciplinaire HAL, est destinée au dépôt et à la diffusion de documents scientifiques de niveau recherche, publiés ou non, émanant des établissements d'enseignement et de recherche français ou étrangers, des laboratoires publics ou privés. 


\title{
Selection Rules for Point-Defect Anelastic Relaxations: Classical versus Quantum Mechanical*
}

\author{
W. Johnson
}

Materials Reliability Division, National Institute of Standards and Technology, 325 Broadway, Boulder, CO 80303, U.S.A.

\begin{abstract}
A quantum mechanical analysis of selection rules for point-defect relaxations under an applied stress yields different results than a conventional classical analysis. The difference in the two approaches is illustrated using a simple 4-well model. The classical selection rules are a special case of the quantum mechanical selection rules with the wavefunctions of the reorienting defect atoms highly localized in potential minima, such that off-diagonal matrix elements of the stress perturbation are negligible.
\end{abstract}

\section{INTRODUCTION}

Selection rules for anelastic relaxations of point defects in crystals can be derived either classically [1-5] or quantum mechanically [6]. Almost all studies use classical selection rules. The relatively few studies that treat anelastic relaxations from a fully quantum mechanical perspective focus on point defects in semiconductors where classical descriptions of the defect states are clearly inadequate (see, for example, Refs. 7-9). The results of the classical and quantum mechanical approaches differ for some defect symmetries. As described in this report, these differences arise from the inability of classical analyses to consider the finite spatial extent of wavefunctions and the resulting neglect of off-diagonal matrix elements of the stress perturbation.

\section{CLASSICAL SELECTION RULES}

Perhaps the simplest model for illustrating the difference between classical and quantum mechanical selection rules is an atom in a tetragonal 4-well potential. Consider an impurity atom $\mathrm{A}$ at a tetragonal $\left(D_{4 h}\right)$ site in a crystal lattice (e.g., centered between next-nearest neighbors in a BCC lattice) and a second atom $B$ trapped near atom $A$ in the plane with four-fold rotational symmetry, as shown in Fig. 1. Atom A is considered to be immobile at the temperatures of interest. Classically, atom $\mathrm{B}$ is localized in one of four equivalent potential wells $(a, b, c$ or $d)$ indicated by dashed circles in Fig. 1 and can be thermally excited from one well to another.

If the crystal lattice is elastically distorted by external stress, the four potential minima may become nonequivalent, depending on the symmetry of the distortion. Two types of pure-shear elastic strain (Fig. 2) are considered here. One, $\epsilon_{2}$, consists of an extension along $\hat{x}$ and a compression along $\hat{y}$, such that the unit cell does not change volume. The other, $\epsilon_{3}$, consists of an extension along $\hat{x}+\hat{y}$ and a compression along $\hat{x}-\hat{y}$ with no change in volume.

The classical selection rules of the defect under $\epsilon_{2}$ and $\epsilon_{3}$ can be determined by inspection of Figs. 1 and 2. Under $\epsilon_{2}$, the four potential minima of atom $B$ remain energetically equivalent. Under $\epsilon_{3}$, minima $a$ and $c$ become different than $b$ and $d$, so that an ensemble of similar defects in a crystal undergoes a thermally activated repopulation between the potential minima, and a corresponding anelastic strain relaxation occurs.

\footnotetext{
* Contribution of NIST. Not subject to copyright in the United States.
} 


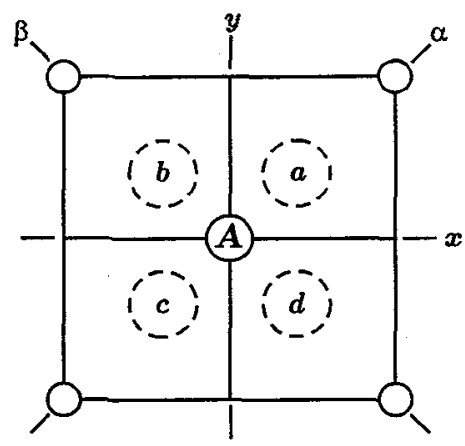

Figure 1: Model defect with atom $A$ at a $D_{4 h}$ site and, classically, atom $B$ (not shown) localized in one of the wells $a, b, c$, or $d$.
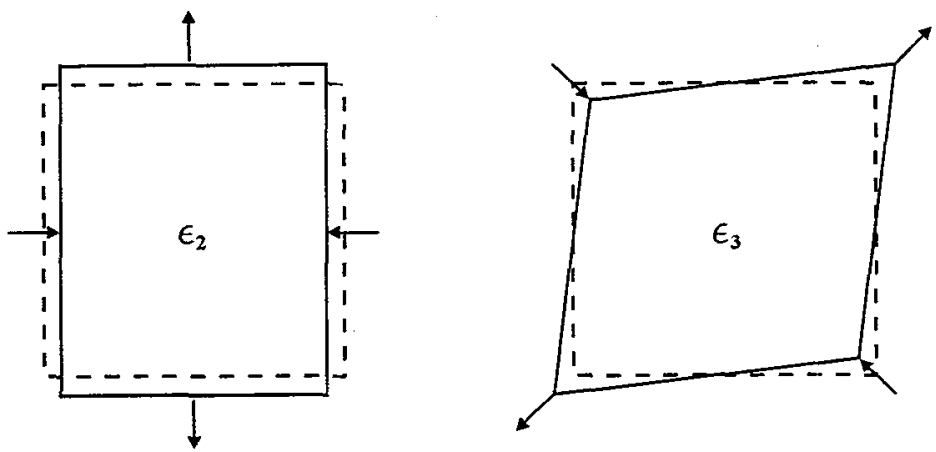

Figure 2: Shear strains.

\section{QUANTUM MECHANICAL SELECTION RULES}

Viewed from a quantum mechanical perspective, the states of atom $\mathrm{B}$ could have wavefunctions $\phi_{a}, \phi_{b}, \phi_{c}$, and $\phi_{d}$ highly localized in the four potential wells $a, b, c$, and $d$, respectively, so that a classical derivation of the selection rules would be valid. If this were the case, the four wavefunctions would be orthogonal because of their lack of overlap, but not because of their symmetry. The fact that such states are not orthogonal on the basis of symmetry can be immediately deduced from the group-theoretical character table of $D_{4 h}$ (Table A.1 in the Appendix), which shows the highest degeneracy to be two-fold.

Table 1: Character table for $\phi_{a}-\phi_{d}$. Definitions of the symmetry operations are given in the Appendix.

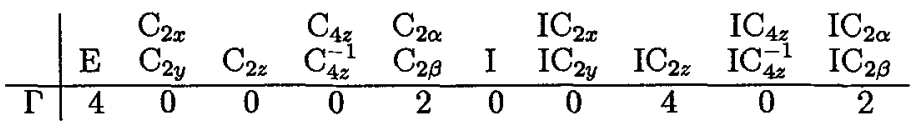

The characters of the reducible representation $\Gamma$ formed by $\left\{\phi_{a} \ldots \phi_{d}\right\}$ are given in Table 1 . This representation reduces to $\Gamma^{1} \oplus \Gamma^{3} \oplus \Gamma^{10}$ with symmetrized basis wavefunctions given in terms of $\phi_{a}-\phi_{d}$ by

$$
\begin{aligned}
\psi^{1} & =4^{-1 / 2}\left[\phi_{a}+\phi_{b}+\phi_{c}+\phi_{d}\right] \\
\psi^{3} & =4^{-1 / 2}\left[\phi_{a}-\phi_{b}+\phi_{c}-\phi_{d}\right] \\
\psi_{1}^{10} & =4^{-1 / 2}\left[\phi_{a}-\phi_{b}-\phi_{c}+\phi_{d}\right] \\
\psi_{2}^{10} & =4^{-1 / 2}\left[\phi_{a}+\phi_{b}-\phi_{c}-\phi_{d}\right]
\end{aligned}
$$


where the superscripts indicate the irreducible representations to which the wavefunctions belong. With negligible overlap of $\left\{\phi_{a} \ldots \phi_{d}\right\}$, the four symmetrized states have the same energy in the undistorted lattice. However, with finite overlap, $\psi^{1}$ and $\psi^{3}$ are nondegenerate (barring further accidental degeneracies), and $\psi_{1}^{10}$ and $\psi_{2}^{10}$ are degenerate. Therefore, if $\left\{\phi_{a} \ldots \phi_{d}\right\}$ are allowed to overlap, the energy level of $\psi_{1}^{10}$ and $\psi_{2}^{10}$ is the only one that can be split by an applied stress. Because of this, $\psi_{1}^{10}$ and $\psi_{2}^{10}$ are the focus here in considering selection rules.

Quantum mechanical selection rules for the model defect can be determined from group theory alone. A brief summary of the group theoretical analysis is presented in the Appendix. The strains $\epsilon_{2}$ and $\epsilon_{3}$ introduce perturbations to the Hamiltonian for atom $\mathrm{B}, H_{2}^{\prime}$ and $H_{3}^{\prime}$, that transform according to $\Gamma^{2}$ and $\Gamma^{3}$, respectively. With both of these perturbations, the degeneracy of $\psi_{1}^{10}$ and $\psi_{2}^{10}$ is removed. Therefore, the quantum mechanical selection rules disagree with the classical selection rules with respect to the effect of $\epsilon_{2}$. The quantum analysis indicates that $\epsilon_{2}$ can split the energy levels; the classical analysis forbids this splitting.

The source of this difference can be understood by considering the perturbing Hamiltonian in the $\left\{\phi_{a} \ldots \phi_{d}\right\}$ basis. With no overlap, the diagonal matrix elements of $H_{2}^{\prime}$ correspond to the classical shifts in energy of the four states (the same for all four states), and the off-diagonal matrix elements are zero:

$$
H_{2}^{\prime} \sim \epsilon_{o}\left(\begin{array}{cccc}
U & 0 & 0 & 0 \\
0 & U & 0 & 0 \\
0 & 0 & U & 0 \\
0 & 0 & 0 & U
\end{array}\right)
$$

where $\epsilon_{\circ}$ is the magnitude of the strain and $U$ is a constant. If this were the exact perturbation, $\psi_{1}^{10}$ and $\psi_{2}^{10}$ would not be split.

If $\left\{\phi_{a} \ldots \phi_{d}\right\}$ are allowed to have finite overlap between adjacent potential wells, some of the offdiagonal matrix elements will be nonzero. From an inspection of Figs. 1 and 2 and a consideration of the symmetries with respect to the reversal of the sign of the strain, the perturbation in the $\left\{\phi_{a} \ldots \phi_{d}\right\}$ basis is found to be

$$
H_{2}^{\prime} \sim \epsilon_{o}\left(\begin{array}{cccc}
V & W & 0 & -W \\
W & V & -W & 0 \\
0 & -W & V & W \\
-W & 0 & W & V
\end{array}\right)
$$

where $V$ and $W$ are constants and $W$ is assumed to be real. The matrix elements between $\psi_{1}^{10}$ and $\psi_{2}^{10}$ are

$$
\begin{aligned}
\left\langle\psi_{1}^{10}\left|H_{2}^{\prime}\right| \psi_{1}^{10}\right\rangle & =\epsilon_{o}(V-2 W) \\
\left\langle\psi_{2}^{10}\left|H_{2}^{\prime}\right| \psi_{2}^{10}\right\rangle & =\epsilon_{o}(V+2 W) \\
\left\langle\psi_{1}^{10}\left|H_{2}^{\prime}\right| \psi_{2}^{10}\right\rangle & =0 \\
\left\langle\psi_{2}^{10}\left|H_{2}^{\prime}\right| \psi_{1}^{10}\right\rangle & =0
\end{aligned}
$$

The degeneracy of $\psi_{1}^{10}$ and $\psi_{2}^{10}$ is removed by $\epsilon_{2}$. The difference between this result and the classical result arises from the neglect of off-diagonal matrix elements $(W)$ in the classical model.

Even though $\epsilon_{2}$ is found to produce anelastic relaxation on the basis of pure symmetry arguments, this relaxation may be negligible compared to that produced by $\epsilon_{3}$. An analysis of the relative significance of the off-diagonal matrix elements is beyond the scope of this paper. One qualitative feature that should be noted is that the rate of quantum tunneling between adjacent potential minima decreases rapidly with increasing barrier height and increasing mass of the tunneling atom. Therefore, classical selection rules will be valid for relaxations involving activation energies and defect masses that are sufficiently large.

\section{Acknowledgement}

The author is grateful to Dr. Andrew Granato for many useful discussions on the subject of selection rules. 


\section{Appendix}

The character table for $D_{4 h}$ is given in Table A.1. The notation used for the representations and symmetry operations follows that given by Cornwell [10], except that the symbols $\alpha$ and $\beta$ are used for the diagonal symmetry axes (Fig. 1) that Cornwell denotes by $a$ and $b$. E is the identity operation. $\mathrm{I}$ is the inversion operation. $\mathrm{C}_{2 x}, \mathrm{C}_{2 y}, \mathrm{C}_{2 \alpha}$, and $\mathrm{C}_{2 \beta}$ are two-fold rotations about the $x, y, \alpha$, and $\beta$ axes, respectively. $\mathrm{C}_{2 z}$ and $\mathrm{C}_{4 z}$ are two-fold and four-fold rotations about the axis perpendicular to the plane of Fig. 1.

Table A.1: Character table for $\mathrm{D}_{4 h}$.

\begin{tabular}{l|l|rrrrrrrrrr} 
& & & $\begin{array}{c}\mathrm{C}_{2 x} \\
\mathrm{C}_{2 y}\end{array}$ & $\mathrm{C}_{2 z}$ & $\begin{array}{c}\mathrm{C}_{4 z} \\
\mathrm{C}_{4 z}^{-1}\end{array}$ & $\begin{array}{c}\mathrm{C}_{2 \alpha} \\
\mathrm{C}_{2 \beta}\end{array}$ & $\mathrm{I}$ & $\begin{array}{r}\mathrm{IC}_{2 x} \\
\mathrm{IC}_{2 y}\end{array}$ & $\mathrm{IC}_{2 z}$ & $\mathrm{IC}_{4 z}^{-1}$ & $\mathrm{IC}_{2 \beta}$ \\
\hline$\Gamma^{1}$ & $\mathrm{~A}_{1 g}$ & 1 & 1 & 1 & 1 & 1 & 1 & 1 & 1 & 1 & 1 \\
$\Gamma^{2}$ & $\mathrm{~B}_{1 g}$ & 1 & 1 & 1 & -1 & -1 & 1 & 1 & 1 & -1 & -1 \\
$\Gamma^{3}$ & $\mathrm{~B}_{2 g}$ & 1 & -1 & 1 & -1 & 1 & 1 & -1 & 1 & -1 & 1 \\
$\Gamma^{4}$ & $\mathrm{~A}_{2 g}$ & 1 & -1 & 1 & 1 & -1 & 1 & -1 & 1 & 1 & -1 \\
$\Gamma^{5}$ & $\mathrm{E}_{g}$ & 2 & 0 & -2 & 0 & 0 & 2 & 0 & -2 & 0 & 0 \\
$\Gamma^{6}$ & $\mathrm{~A}_{1 u}$ & 1 & 1 & 1 & 1 & 1 & -1 & -1 & -1 & -1 & -1 \\
$\Gamma^{7}$ & $\mathrm{~B}_{1 u}$ & 1 & 1 & 1 & -1 & -1 & -1 & -1 & -1 & 1 & 1 \\
$\Gamma^{8}$ & $\mathrm{~B}_{2 u}$ & 1 & -1 & 1 & -1 & 1 & -1 & 1 & -1 & 1 & -1 \\
$\Gamma^{9}$ & $\mathrm{~A}_{2 u}$ & 1 & -1 & 1 & 1 & -1 & -1 & 1 & -1 & -1 & 1 \\
$\Gamma^{10}$ & $\mathrm{E}_{u}$ & 2 & 0 & -2 & 0 & 0 & -2 & 0 & 2 & 0 & 0
\end{tabular}

Table A.2: Notations of Cornwell [10] and Koster et al. [11] for the irreducible representations of $D_{4 h}$.

\begin{tabular}{l|llllllllll} 
Cornwell & $\Gamma^{1}$ & $\Gamma^{2}$ & $\Gamma^{3}$ & $\Gamma^{4}$ & $\Gamma^{5}$ & $\Gamma^{6}$ & $\Gamma^{7}$ & $\Gamma^{8}$ & $\Gamma^{9}$ & $\Gamma^{10}$ \\
\hline Koster et al. & $\Gamma_{1}^{+}$ & $\Gamma_{3}^{+}$ & $\Gamma_{4}^{+}$ & $\Gamma_{2}^{+}$ & $\Gamma_{5}^{+}$ & $\Gamma_{1}^{-}$ & $\Gamma_{3}^{-}$ & $\Gamma_{4}^{-}$ & $\Gamma_{2}^{-}$ & $\Gamma_{5}^{-}$
\end{tabular}

The tables of coupling coefficients presented by Koster et al. [11] are used to determine selection rules. Unfortunately, the numbering used by Koster et al. for the irreducible representations of $\mathrm{D}_{4 h}$ does not match that of Cornwell [10]. Table A.2 gives the translation between the notations of these two references. A detailed description of the use of the tables of Koster et al. is beyond the scope of this paper. However, for the symmetries of the states and strains of interest here, the procedure is relatively simple. The perturbations $H_{2}^{\prime}$ and $H_{3}^{\prime}$ in the $\left\{\psi_{1}^{10}, \psi_{2}^{10}\right\}$ basis have the same form as the corresponding matrices of coupling coefficients within a similarity transformation and a multiplicative constant. In the diagonalized $\left\{\psi_{1}^{10}, \psi_{2}^{10}\right\}$ basis, the perturbation from $\epsilon_{2}$ is found to have matrix elements $C_{2}$ and $-C_{2}$, where $C_{2}$ is a constant. This is the same result as that presented in Eqs. (7)-(10), except that the group theoretical analysis provides the additional information that $V$ must be 0 . Similarly, the diagonalized perturbation from $\epsilon_{3}$ has matrix elements $C_{3}$ and $-C_{3}$, where $C_{3}$ is a second constant. Classical selection rules will be valid if $C_{2}$ is much less than $C_{3}$.

\section{References}

[1] A. S. Nowick and W. R. Heller, Advan. Phys. 12 (1963) 251-298.

[2] A. S. Nowick and W. R. Heller, Advan. Phys. 14 (1965) 101-166.

[3] J. B. Wachtman, Jr., and H. S. Peiser, Appl. Phys. Lett. 1 (1962) 20-22.

[4] H. S. Peiser and J. B. Wachtman, Jr., J. Res. NBS-A 69A (1965) 309-324.

5] J. B. Wachtman, Jr., and H. S. Peiser, J. Phys. Chem. Solids 27 (1966) 975-982.

[6] W. L. Johnson, Ph.D. thesis, Physics Department, Univ. of Ill. at Urbana-Champaign (University Microfilms Int., no. DA8711816) (1987).

[7] G. L. Bir, E. I. Butikov, and G. E. Pikus, J. Phys. Chem. Solids 24 (1963) 1467-1474.

[8] Y. Isawa, Y. Takeuti, and N. Mikoshiba, Phys. Rev. B 15 (1977) 4907-4922.

[9] T. Miyasato, M. Tokumura, M. Toguchi, and F. Akao, J. Phys. Soc. Jap. 49 (1980) 2219-2226.

[10] J. F. Cornwell, Group Theory in Physics (Academic, New York, 1984), Vol. 1.

[11] G. F. Koster, J. O. Dimmock, R. G. Wheeler, and H. Statz, Properties of the Thirty-two Point Groups (M.I.T., Cambridge, 1963). 